\title{
Should early extubation be the goal for children after congenital cardiac surgery?
}

\author{
Kevin C. Harris, MD, MHSc, ${ }^{a}$ Spencer Holowachuk, BHK, ${ }^{a}$ Sandy Pitfield, MD, ${ }^{b}$ \\ Shubhayan Sanatani, MD, ${ }^{\mathrm{a}}$ Norbert Froese, MD, ${ }^{\mathrm{c}}$ James E. Potts, PhD, ${ }^{\mathrm{a}}$ and Sanjiv K. Gandhi, MD ${ }^{\mathrm{d}}$
}

Objective: We sought to determine the feasibility and assess the clinical outcomes associated with an early extubation strategy for all children undergoing congenital heart surgery, including neonates (age, $<30$ days).

\begin{abstract}
Methods: We performed a linked database analysis of all patients undergoing congenital heart surgery from July 1, 2010 to December 31, 2012. We collected data on the cardiac diagnoses, preoperative status, procedure, and postoperative course, including the duration of invasive and noninvasive ventilation, failure of extubation, hemodynamic data, length of stay, complications, and mortality. A multivariable model was used to assess the independent factors associated with an inability to extubate within the operating room and with delayed extubation ( $>24$ hours).
\end{abstract}

Results: We operated on 613 children, including 97 neonates. Intraoperative extubation was achieved in $71 \%$ of the cases and early extubation ( $\leq 24$ hours) was achieved in $89 \%$ of the cases. The overall mortality was $1.5 \%$ ( 9 of 613 patients). Early extubation was associated with lower mortality $(1 \%$ vs $9 \%, P<.001)$ and a lower rate of reintubation ( $4 \%$ vs $23 \%, P<.001$ ) compared with delayed extubation. Notably, $63 \%$ of the neonates were extubated within 24 hours, including $67 \%$ of arterial switch operations and $54 \%$ of total anomalous pulmonary venous return repairs. Norwood operations were the only procedure in which no patient was extubated within the first 24 hours. Multivariable logistic regression demonstrated that the predictors of delayed extubation included preoperative mechanical ventilation, weight $<5 \mathrm{~kg}$, a longer procedure time, and the need for postoperative inotrope support. Implementation of an early extubation strategy was associated with low rates of complications (5.1 per 10 procedures), short lengths of intensive care unit stay (median, 1 day; interquartile range, 1-3), and short hospital stays (median, 4 days; interquartile range, 3-6).

Conclusions: Most children undergoing congenital heart surgery can be extubated in the operating room. Most neonates, including many undergoing complex procedures, can be extubated within the first 24 hours after surgery. Early extubation was associated with low morbidity rates and short lengths of intensive care unit and hospital stays. (J Thorac Cardiovasc Surg 2014;148:2642-8)

See related commentary on pages 2649-50.

The need for prolonged mechanical ventilation for children with congenital heart disease after cardiac surgery has changed with the advances in surgical and perioperative care. Early extubation (EE), albeit variably defined in published studies (within the operating room, $\leq 6$ hours

\footnotetext{
From the Divisions of Cardiology, ${ }^{\mathrm{a}}$ Critical Care, ${ }^{\mathrm{b}}$ and Cardiac Surgery, ${ }^{\mathrm{d}}$ and Department of Anesthesia, ${ }^{\mathrm{c}}$ British Columbia Children's Hospital, Vancouver, British Columbia, Canada.

Disclosures: Authors have nothing to disclose with regard to commercial support.

Read at the 94th Annual Meeting of The American Association for Thoracic Surgery, Toronto, Ontario, Canada, April 26-30, 2014.

Received for publication April 10, 2014; revisions received June 10, 2014; accepted for publication June 27, 2014; available ahead of print Aug 22, 2014.

Address for reprints: Kevin C. Harris, MD, MHSc, Division of Cardiology, British Columbia Children's Hospital, Vancouver, BC V6H3V4, Canada (E-mail: kharris2@cw.bc.ca).

$0022-5223 / \$ 36.00$

Copyright (c) 2014 by The American Association for Thoracic Surgery

http://dx.doi.org/10.1016/j.jtcvs.2014.06.093
}

postoperatively, or $\leq 24$ hours postoperatively), has been implemented as a part of the management strategy for children undergoing cardiac surgery. ${ }^{1}$ This has been associated with improved resource usage by shortening the intensive care unit (ICU) and hospital lengths of stay in a range of patients with congenital heart disease. ${ }^{2,3}$ Many of the early studies have demonstrated the feasibility of this approach in select infants and older children. In part owing to the selected nature of the populations, the number of children included in these studies was often small. ${ }^{4-6}$ Despite the positive findings of these early studies, early extubation strategies have not been rigorously evaluated when applied to an unselected population of pediatric patients undergoing congenital heart surgery. In particular, very little data have been published on the feasibility, clinical outcomes, or resource usage associated with early extubation in neonates or infants undergoing complex cardiac surgery. This might be, in part, because previous studies have shown that the strongest independent risk factors for failure of EE are younger age and longer cardiopulmonary bypass times. ${ }^{6}$ 


$$
\begin{aligned}
& \text { Abbreviations and Acronyms } \\
& \begin{aligned}
\mathrm{BT} & =\text { Blalock-Taussig } \\
\mathrm{DE} & =\text { delayed extubation }(>24 \text { hours of arrival in ICU) } \\
\mathrm{EE} & =\text { early extubation }(\leq 24 \text { hours of arrival in ICU }) \\
\mathrm{ICU} & =\text { intensive care unit } \\
\mathrm{IE} & =\text { intraoperative extubation } \\
\mathrm{IQR} & =\text { interquartile range }
\end{aligned}
\end{aligned}
$$

The goal of our study was to determine the feasibility and assess the clinical effect of implementing an early extubation strategy for all children undergoing congenital heart surgery, including neonates $(<30$ days) and those with complex cardiac malformations. We sought to determine the factors associated with successful EE. We reviewed the survival, perioperative complication rates, and length of stay to evaluate the success of EE in this population.

\section{METHODS}

We performed a linked database analysis of all patients undergoing congenital heart surgery at British Columbia Children's Hospital from July 1, 2010 to December 31, 2012. All patients undergoing congenital cardiac surgical procedures were included in the analysis, with the exception of premature neonates, who underwent isolated patent ductus arteriosus ligation. We also excluded children with preoperative tracheostomy associated long-term mechanical ventilation dependence.

Our institutional approach was to extubate every patient as soon as possible after surgery. The candidacy of each patient for EE was discussed between the surgeon and anesthesiologist before surgery and again after surgery (taking into account the operative course, transesophageal echocardiographic findings, and overall cardiorespiratory status of the patient). If the patient remained intubated after surgery, the need for ongoing mechanical ventilation was assessed by a multidisciplinary team involving cardiac surgery, cardiology, and ICU at least twice every 24 hours.

\section{Perioperative Management}

Non-neonatal patients were premedicated with oral midazolam (0.5-0.8 $\mathrm{mg} / \mathrm{kg}$ ) and acetaminophen. Anesthesia was induced either by mask inhalation of sevoflurane or intravenous propofol and/or ketamine. Rocuronium was administered to facilitate tracheal intubation and repeated only if indicated. Anesthesia was maintained with bolus doses of intravenous fentanyl and inhaled isoflurane. Most patients received an infusion of dexmedetomidine $(0.6 \mu \mathrm{g} / \mathrm{kg} / \mathrm{h})$ for the duration of the case. Spontaneous ventilation was achieved after sternal approximation in patients suitable for operating room extubation. Muscle relaxant reversal agents were administered as required. Additional fentanyl was administered, titrated to effect. Patients achieving a stable respiratory pattern were extubated in the operating room deeply sedated and transferred to the ICU.

\section{ICU Management}

ICU management of intraoperatively extubated patients focused on maintaining adequate analgesia and sedation without depressing cardiorespiratory function. This was accomplished in most patients with modest doses of dexmedetomidine $(0.1-0.7 \mu \mathrm{g} / \mathrm{kg} / \mathrm{h})$ and morphine $(0-20 \mu \mathrm{g} / \mathrm{kg} / \mathrm{h})$ or fentanyl $(0-2 \mu \mathrm{g} / \mathrm{kg} / \mathrm{h})$. Supplementary anxiolysis was occasionally needed using chloral hydrate $(10-15 \mathrm{mg} / \mathrm{kg} / \mathrm{dose})$. The patients who were not extubated before ICU admission were screened at admission to the ICU regarding candidacy for early postoperative extubation. Those patients with stable cardiac function and reasonable respiratory system function received minimal additional analgesics or anxiolytics (dexmedetomidine $0.2 \mu \mathrm{g} / \mathrm{kg} / \mathrm{h}$ and morphine $5 \mu \mathrm{g} / \mathrm{kg} / \mathrm{h}$ ) until the level of consciousness was compatible with extubation. After extubation, excluding patients at risk of gastric reperfusion injury, most patients were offered sips of fluids to provide comfort.

\section{Data Collection}

The hospital course of all patients were evaluated using linked data from the ICU, cardiac surgery, cardiac perfusion, and cardiology databases and patient medical record review. All databases were maintained prospectively, with the data entered at the clinical encounter. The cardiac surgery database was maintained by the cardiac surgeons and included the preoperative diagnosis and surgical procedure, which were recorded immediately after the procedure. The intraoperative variables, including cardiopulmonary bypass time, crossclamp time, and circulatory arrest time, were collected prospectively and documented in the cardiac perfusion database. The ICU database included the data on hemodynamic variables, ventilator support, complications, and length of stay. The data were collected by a nurse coordinator on a daily basis from medical record review and in consultation with the treating physicians when the medical record data were incomplete. The cardiology database contained data on the postoperative complications with data entered daily in real-time using a portable handheld device at the bedside by the attending cardiologist. The databases were linked using administrative data that were shared among the databases.

We collected data on the cardiac diagnoses, preoperative status, surgical procedure, and postoperative course, including the duration of invasive and noninvasive ventilation, failure of extubation, hemodynamic data, length of stay, complications, and mortality. A detailed patient medical record review was performed for those children who were admitted to the ICU preoperatively requiring ventilatory support, those admitted to the ICU intubated postoperatively, those who, after extubation, required reintubation, and any other patients in whom the duration of mechanical ventilation was not clearly delineated. In such cases, the medical record review was used to determine the length of postoperative intubation through a review of the respiratory therapy and nursing notes. The time of admission to the ICU was recorded as the postoperative intubation start time, and the times of extubation and reintubation (where applicable) were recorded.

We categorized patients according to the extubation time. We defined intraoperative extubation (IE) as those patients who were extubated before transfer from the operating room to the ICU. We defined EE as an initial extubation within 24 hours of arrival to the ICU. We defined delayed extubation (DE) as an initial extubation $>24$ hours from the postoperative arrival in the ICU. Children who required reintubation were allocated according to their initial time of extubation, and all analyses were conducted according to this initial group allocation.

\section{Statistical Analysis}

Frequency tables were generated for all categorical variables, and the data are presented as the number and/or percentage of patients. Univariate analysis was performed on all continuous variables. The data are reported as the median and interquartile range (IQR). The data were categorized for patients by (1) age at surgery ( $<1$ month, 1-2, 2-4, 4-6, 6-12, and $>12$ months) and (2) the timing of extubation (IE, EE, and DE). Frequency histograms were generated for the partial pressure of oxygen and carbon dioxide in arterial blood, cardiopulmonary bypass time, and circulatory arrest time. The median value was used to create dichotomized groups. Group differences for all categorical data were compared using a 
chi-square test and for all continuous data using a Wilcoxon signed-rank test.

Adjusted odds ratios with $95 \%$ confidence intervals were estimated for independent variables associated with the timing of extubation using a multivariable stepwise logistic regression procedure. The independent variables included sex, age, weight, prematurity, chromosomal abnormality, cardiac diagnosis, surgical procedure, preoperative mechanical ventilation, noninvasive ventilator support (bilevel positive airway pressure, continuous positive airway pressure, or synchronized inspiratory positive airway pressure), cardiopulmonary bypass time, circulatory arrest time, lowest partial pressure of oxygen in arterial blood, lowest hemoglobin, and highest partial pressure of carbon dioxide in arterial blood. This model was derived from the best fit of our data set and, therefore, might not be applicable to other data sets. The complication rates are reported using standardized definitions outlined by the Multi-Societal Committee for Pediatric and Congenital Heart Disease. ${ }^{7}$ A 2 -sided $P$ value $<.05$ was considered statistically significant. All data were analyzed using the Statistical Analysis Systems statistical software, version 9.3 (SAS Institute, Cary, NC).

The University of British Columbia Children's and Women's Health Centre's Clinical Research Ethics Board approved the study.

\section{RESULTS}

We identified 617 patients who underwent cardiac surgery and were admitted to the ICU postoperatively. We excluded 4 children who, preoperatively, had received a tracheostomy and were dependent on longterm mechanical ventilation. We performed a detailed review of the remaining 613 children.

\section{Demographics}

Our cohort consisted of 613 patients, 337 of whom were male $(55 \%)$. Of the 613 patients, $97(16 \%)$ were neonates (age, $<30$ days old), 30 were 1 to 2 months old (5\%), 73 were 2 to 4 months old (12\%), 84 were 4 to 6 months old $(14 \%), 56$ were 6 to 12 months old $(9 \%)$, and 273 were $>12$ months old $(44 \%)$ at surgery. Of the 613 patients, 69 $(11 \%)$ had chromosomal abnormalities, including 52 with trisomy 21, 12 with chromosomal microdeletions, 1 each with trisomy 22 and mosaic trisomy 13, and 2 with other genetic anomalies. Of the 613 patients, $19(3 \%)$ were born prematurely (gestational age, $<37$ weeks).

Before surgery, 54 of the 613 patients $(9 \%)$ were receiving inotropic support, 22 patients $(4 \%)$ were receiving short-term mechanical ventilation, and 5 patients $(1 \%)$ required noninvasive respiratory support with bilevel positive airway pressure, continuous positive airway pressure, or synchronized inspiratory positive airway pressure.

\section{Procedural Details}

Of the 613 surgical cases, 535 were open heart $(87 \%)$ and 78 were closed heart procedures $(13 \%)$. The median patient age at surgery was 7.1 months (IQR, 2.9-57.4; Figure 1), and median weight was $7.2 \mathrm{~kg}$ (IQR, 4.417.0). The complexity of each case was assessed using the Risk Adjustment for Congenital Heart Surgery score. ${ }^{8}$

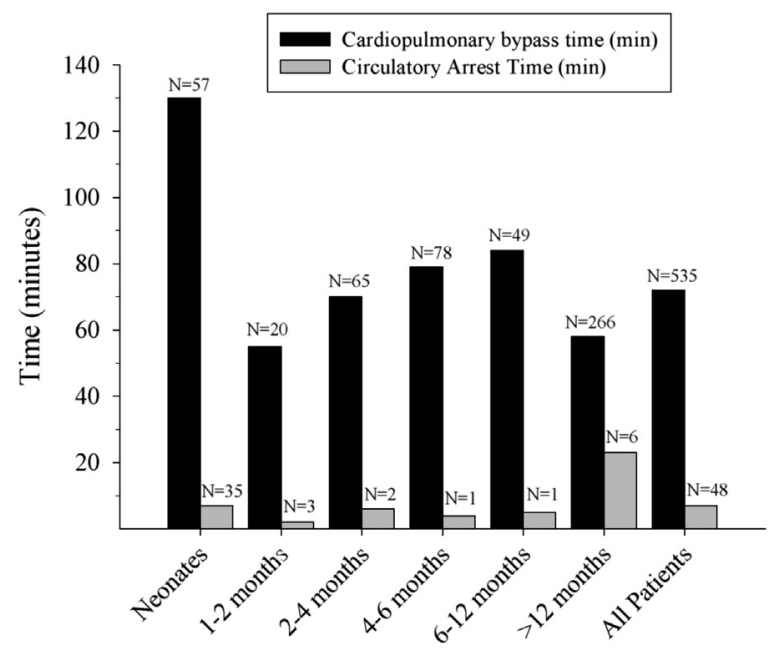

FIGURE 1. Cardiopulmonary bypass and circulatory arrest times stratified by age group (<30 days and 1-2, 2-4, 4-6, 6-12, and $>12$ months) and the number of subjects in each cohort.

Of the 613 patients, $117(19 \%)$ were assigned to risk category $1 ; 247(41 \%)$ to risk category $2 ; 220(36 \%)$ to risk category $3 ; 21(3 \%)$ to risk category 4 ; and $8(1 \%)$ to risk category 6 .

The median cardiopulmonary bypass and crossclamp time during the 535 pump cases was 72 minutes (IQR, 47-110) and 36 minutes (IQR, 21-66), respectively. Circulatory arrest was required in 48 patients. The median circulatory arrest time in these patients was 7 minutes (IQR, 4.5-14.0). Figure 1 illustrates the median bypass and circulatory arrest times stratified by age group. The cardiopulmonary bypass (median, 130 vs 64 minutes; $P<.001$ ) and crossclamp (median, 77.5 vs 33 minutes; $P<.001)$ times were considerably longer for the neonates compared with all other patients. No difference was found in the circulatory arrest times (median, 7 vs 5 minutes; $P=.370$ ) between the neonates and the older cohort of patients.

\section{Early Versus Late Extubation}

IE was achieved in 437 of 613 patients $(71 \%)$, and 548 patients $(89 \%)$ were extubated within 24 hours (EE). Extubation was delayed ( $>24$ hours, DE) in the remaining 65 patients $(11 \%)$. The point at which the patients were extubated and the number and percentage of patients who were extubated early for each surgical procedure are listed in Table 1. IE was achieved in 16 of 97 neonates $(16 \%)$, and 61 neonates $(63 \%)$ were extubated within 24 hours. EE was achieved in many neonates undergoing higher risk procedures, including the arterial switch operation $(67 \%$ overall; $86 \%$ for simple transposition of the great arteries) and neonatal total anomalous pulmonary venous return repairs $(54 \%)$. Norwood operations were the only 
TABLE 1. Procedures performed and percentage of patients extubated early

\begin{tabular}{|c|c|c|c|c|c|c|}
\hline \multirow[b]{2}{*}{ Procedure } & \multicolumn{4}{|c|}{ Extubation time (n) } & \multicolumn{2}{|c|}{ EE rate $(\%)$} \\
\hline & OR & 0-24 h & $>24 \mathrm{~h}$ & Total & OR & $\leq \mathbf{2 4 h}$ \\
\hline ASD repair & 89 & 1 & 0 & 90 & $89 / 90(99)$ & $90 / 90(100)$ \\
\hline ASO & 2 & 16 & 9 & 27 & $2 / 27(7)$ & $18 / 27(67)$ \\
\hline AVSD repair & 17 & 12 & 1 & 30 & $17 / 30(57)$ & 29/30 (97) \\
\hline LVOT surgery & 22 & 1 & 1 & 24 & $22 / 24(92)$ & $23 / 24(96)$ \\
\hline $\mathrm{IAA} / \mathrm{CoA}+\mathrm{VSD}$ & 0 & 2 & 3 & 5 & $0 / 5(0)$ & $2 / 5(40)$ \\
\hline $\mathrm{CoA}$ & 26 & 13 & 3 & 42 & $26 / 42(62)$ & $39 / 42(93)$ \\
\hline Fontan procedure & 22 & 0 & 0 & 22 & $22 / 22(100)$ & $22 / 22(100)$ \\
\hline Glenn operation & 25 & 3 & 0 & 28 & 25/28 (89) & $28 / 28(100)$ \\
\hline MV & 10 & 3 & 0 & 13 & 10/13 (77) & $13 / 13(100)$ \\
\hline $\begin{array}{l}\text { Norwood } \\
\text { procedure }\end{array}$ & 0 & 0 & 8 & 8 & $0 / 8(0)$ & $0 / 8(0)$ \\
\hline PDA ligation & 6 & 1 & 0 & 7 & $6 / 7(86)$ & 7/7 (100) \\
\hline RV-PA conduit & 45 & 4 & 4 & 53 & $45 / 53(85)$ & $49 / 53(93)$ \\
\hline Ross procedure & 8 & 2 & 1 & 11 & 8/11 (72) & 10/11 (91) \\
\hline BT shunt & 9 & 17 & 7 & 33 & 9/33 (27) & $26 / 33$ (79) \\
\hline TAPVR repair & 3 & 4 & 6 & 13 & $3 / 13(23)$ & $7 / 13(54)$ \\
\hline TOF repair & 16 & 8 & 7 & 31 & $16 / 31(52)$ & $24 / 31(77)$ \\
\hline VSD repair & 106 & 18 & 6 & 130 & $106 / 130(82)$ & $124 / 130(95)$ \\
\hline Other & 31 & 6 & 9 & 46 & $31 / 46(67)$ & $37 / 46(80)$ \\
\hline Total & 437 & 111 & 65 & 613 & $437 / 613(71)$ & $548 / 613(89)$ \\
\hline \multicolumn{7}{|c|}{$\begin{array}{l}E E \text {, Early extubation; } O R \text {, operating room; } A S D \text {, atrial septal defect; } A S O \text {, arterial } \\
\text { switch operation; } A V S D \text {, atrioventricular septal defect; } L V O T \text {, left ventricular outflow } \\
\text { tract; } I A A \text {, interrupted aortic arch; } C O A \text {, coarctation of the aorta; } V S D \text {, ventricular } \\
\text { septal defect; } M V \text {, mitral valve; } P D A \text {, patent ductus arteriosus; } R V-P A \text {, right ventricle } \\
\text { to pulmonary artery; } B T \text {, Blalock-Taussig; } T A P V R \text {, total anomalous pulmonary } \\
\text { venous return; } T O F \text {, tetralogy of Fallot. }\end{array}$} \\
\hline
\end{tabular}

procedure in which no patient was extubated within the first 24 hours. The percentage of patients in each age group who was extubated early ( $\leq 24$ hours) is presented in Figure 2.

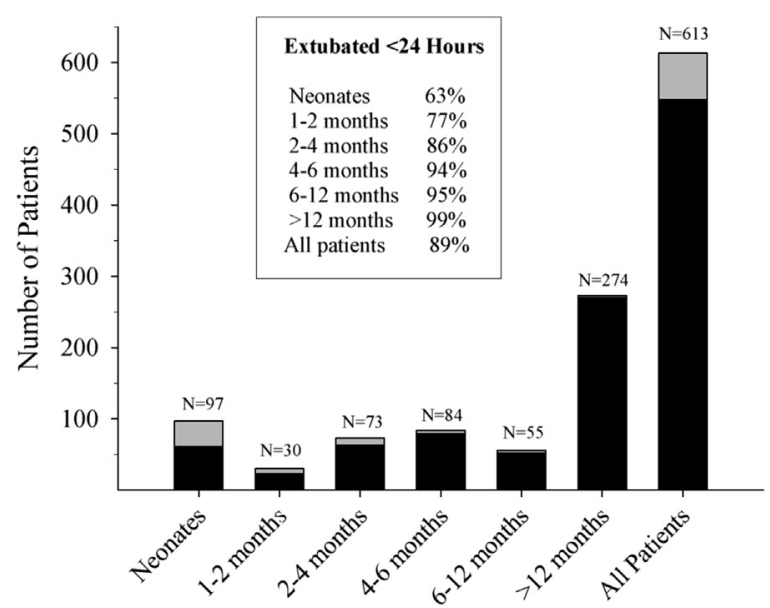

FIGURE 2. Frequency distribution of surgical age sorted into age groups (neonates $[<30$ days $]$ and 1-2, 2-4, 4-6, 6-12, and $>12$ months). The percentage of patients extubated early $(\leq 24$ hours) in each cohort is indicated in the text box and represented in each bar in black.
TABLE 2. Early extubation rates analyzed by age and time

\begin{tabular}{|c|c|c|c|c|c|}
\hline \multirow[b]{2}{*}{ Age } & \multicolumn{3}{|c|}{ EE } & \multirow[b]{2}{*}{ DE $(>24$ h) } & \multirow[b]{2}{*}{ Overall total } \\
\hline & OR & $0-24$ h & Total & & \\
\hline Neonate & $16(17)$ & $45(46)$ & $61(63)$ & $36(37)$ & 97 (100) \\
\hline $1-2 \mathrm{mo}$ & $15(50)$ & $8(27)$ & $23(77)$ & $7(23)$ & $30(100)$ \\
\hline $2-4$ mo & $40(55)$ & $23(31)$ & $63(86)$ & $10(14)$ & 73 (100) \\
\hline 4-6 mo & $60(71)$ & $19(23)$ & 79 (94) & $5(6)$ & $84(100)$ \\
\hline 6-12 mo & $48(86)$ & $5(9)$ & $53(95)$ & $3(5)$ & $56(100)$ \\
\hline$>12 \mathrm{mo}$ & $258(95)$ & $11(4)$ & 269 (99) & $4(1)$ & $273(100)$ \\
\hline Total & $437(71)$ & $111(18)$ & $548(89)$ & $65(11)$ & $613(100)$ \\
\hline
\end{tabular}

Data presented as $\mathrm{n}(\%)$. $E E$, Early extubation; $D E$, delayed extubation; $O R$, operating room.

The EE rates increased with age $(P<.001)$. When the patients were extubated (IE, EE, or DE) stratified by age is listed Table 2. The bypass (median, 63 vs 135 minutes; $P<.001$ ) and crossclamp (median, 32.5 vs 70.5 minutes; $P<.001)$ times were shorter in the EE cohort. The partial pressure of oxygen in arterial blood, recorded in the first 12 postoperative hours, was lower in the DE cohort (median, 51.5 vs $83 \mathrm{~mm} \mathrm{Hg} ; P<.001$ ). The circulatory arrest time (median, 7 vs 8 minutes; $P=.656$ ) was similar in these patient groups.

\section{Reintubation}

The overall reintubation rate was $6 \%$ (35 of 613 ). Of the 548 children extubated early, 20 patients required reintubation $(4 \%)$. Ten patients, including 2 neonates, required reintubation after being extubated in the operating room. These included 3 patients with Blalock-Taussig (BT) shunts, 2 with atrioventricular septal defect repairs, 1 who underwent completion of a Fontan procedure, 1 who underwent a bidirectional Glenn procedure, 1 with a ventricular septal defect repair, 1 with a right ventricle to pulmonary artery valved conduit, and 1 patient who underwent tetralogy of Fallot repair. Ten patients, including 5 neonates, who were extubated within 24 hours of leaving the operating room, needed to be reintubated. These included 6 patients with shunts, 1 with coarctation, 1 with an atrioventricular septal defect repair, 1 with a right ventricle to pulmonary artery valved conduit, and 1 patient with a tetralogy of Fallot repair. In the EE group, the reintubation rate for neonates was $11 \%$ (7 of 61), of whom 6 underwent BT shunt placement. EE was associated with lower rates of reintubation compared with DE (4\% vs $23 \%, P<.001)$.

The predictors of DE determined by the multivariable logistic regression model were the need for preoperative mechanical ventilation, weight $<5 \mathrm{~kg}$, a longer procedure time, and the need for postoperative inotrope support. The individual predictors of failed extubation in the operating room and DE ( $>24$ hours) are listed in Table 3. 
TABLE 3. Predictors of delayed extubation

\begin{tabular}{|c|c|c|c|c|}
\hline \multirow[b]{2}{*}{ Variable } & \multicolumn{2}{|c|}{ Failure to extubate in operating room } & \multicolumn{2}{|c|}{ DE ( $>24$ h) } \\
\hline & OR $(95 \%$ CI $)$ & $P$ value & OR $(95 \%$ CI $)$ & $P$ value \\
\hline Preoperative mechanical ventilation & $14.5(2.3-90.4)$ & .004 & $9.4(3.2-28.2)$ & $<.001$ \\
\hline Weight $\leq 5 \mathrm{~kg}$ & $10.3(5.6-18.9)$ & $<.001$ & $7.4(3.4-16.1)$ & $<.001$ \\
\hline Bypass time $>140 \mathrm{~min}$ & $5.9(2.7-12.8)$ & $<.001$ & $9.5(4.1-21.7)$ & $<.001$ \\
\hline Circulatory arrest time $>7 \mathrm{~min}$ & $16.7(1.9-142.9)$ & .01 & $4.8(1.5-16.1)$ & .01 \\
\hline Postoperative inotrope use & $3.9(2.2-7.0)$ & $<.001$ & $11.5(2.6-50.8)$ & $<.001$ \\
\hline $\mathrm{PaO}_{2}<80 \mathrm{~mm} \mathrm{Hg}$ & $3.3(1.9-5.5)$ & $<.001$ & $4.4(1.9-10.0)$ & $<.001$ \\
\hline Age $\leq 30 \mathrm{~d}$ & $2.9(1.3-6.3)$ & .009 & - & - \\
\hline Male sex & $2.6(1.5-4.3)$ & $<.001$ & - & - \\
\hline
\end{tabular}

$\mathrm{DE}$, Delayed extubation; $\mathrm{OR}$, odds ratio; $\mathrm{CI}$, confidence interval; $\mathrm{PaO}_{2}$, partial pressure of oxygen in arterial blood.

\section{Complications}

The overall complication rate in our cohort was 5.1 complications per 10 procedures, with $27 \%$ of all procedures associated with $\geq 1$ complication. The patients in the EE cohort were less likely to develop postoperative complications $(20 \%$ vs $85 \% ; P<.001)$ and had lower complication rates ( 3 vs 22 complications per 10 procedures; $P<.001$ ). The median length of stay in the ICU and hospital was 1 day (IQR, 1-3) and 4 days (IQR, 3-6), respectively. EE was associated with shorter lengths of stay in the ICU (median, 1 vs 7 days; $P<.001$ ) and hospital (median, 4 vs 11 days; $P<.001$ ).

\section{Mortality}

Overall mortality was $1.5 \%$ (9 of 613). EE was associated with lower mortality compared with DE $(1 \%$ vs $9 \%, P<.001)$. Of the 3 EE patients who died, 2 were extubated in the operating room (1 neonate undergoing a BT shunt and 1, aged $>12$ months, undergoing a Fontan procedure) and 1, aged 2 to 4 months old, was extubated within 24 hours (atrioventricular septal defect). Comparatively, 6 deaths occurred in the DE group: 4 neonates ( 3 Norwood procedures, 1 BT shunt), 1 patient aged 4 to 6 months old (tetralogy of Fallot), and 1 patient aged $>12$ months (right ventricle to pulmonary artery conduit).

\section{DISCUSSION}

Although it is well established that EE is safe and feasible in children undergoing congenital heart surgery, our results have confirmed that this perioperative management approach can be extended to neonates, including select neonates and infants undergoing higher risk surgical repairs. We have demonstrated a high rate of EE in neonates $(63 \%)$, including many neonates undergoing higher risk procedures such as arterial switch operations $(86 \%$ for simple transposition of the great arteries and $67 \%$ for transposition of the great arteries overall) and repair of total anomalous pulmonary venous return $(54 \%)$. The only surgical procedure in which no patient was extubated in the first 24 hours postoperatively was the Norwood operation.

One of the key strengths of the present study was that we included all patients in our analysis. Although we did not attempt EE for any of our neonates who had undergone a Norwood procedure, we did not exclude patients a priori because of age, cardiac diagnosis, or perceived surgical complexity or risk. Previous studies have excluded neonates ${ }^{6,9}$ and infants $<6$ months old. ${ }^{3,10}$ Through inclusion of all children undergoing congenital heart surgery, we have shown that the reintubation rates are low, and, of particular interest, no risk factors for requiring reintubation after EE were identified. The few children who required reintubation were spread across the age groups and primary diagnoses. BT shunt placement was associated with greater reintubation rates in the neonates. It has previously been shown that the clinical predictors of successful extubation are poor and that clinical protocols are likely superior to subjective weaning of support in infants and children. ${ }^{11-13}$ Given the morbidity and known risks associated with intubation and mechanical ventilation, ${ }^{14}$ our institution has developed an approach such that EE is the norm, and patients only remain intubated if a compelling cardiorespiratory benefit is present, regardless of age. This approach has been rapidly adopted and, in the present series, we have confirmed that it is associated with low morbidity and short lengths of stay.

Reducing the length of ICU stay and total hospital stay leads to improved resource utilization. Recent studies have demonstrated that considerable variability exists in the outcomes and costs associated with cardiac surgery for children with congenital heart disease. A significant proportion of the variability in costs observed among institutions has been associated with the variability in complication rates and lengths of stay. ${ }^{15}$ Hence, broad implementation of EE has the potential to reduce the total 
costs associated with pediatric cardiac surgery and reduce the interinstitutional variability.

Compared with published standards, our program has had low mortality and complication rates. The aggregate multi-institutional mortality for pediatric cardiac surgery is $4 \%{ }^{16,17}$ and $\leq 32 \%$ of operations are associated with $\geq 1$ complication. ${ }^{18}$ In contrast, we found a mortality rate of $1.5 \%$ and a complication rate of $27 \%$. Furthermore, our linked database analysis had multiple layers of redundancy to maximize the opportunity for the complete capture of complications in the present study. As the only tertiary care cardiac surgery center in British Columbia, we provide exclusive service to a population of $>4$ million residents, and our case distribution reflects the distribution of cardiac pathologic entities in children in British Columbia. Our morbidity and mortality data provide additional support for $\mathrm{EE}$ as an important perioperative management strategy. Reducing complications further will contribute to optimal resource use in addition to improving clinical outcomes.

Our study was limited by the retrospective nature of the analysis. Given that there was a programmatic implementation of early extubation at our institution, we did not have a contemporary control cohort. We did not believe that a retrospective control group would be valid, given the potential for unmeasurable confounders that can be associated with a programmatic change. Nonetheless, comparing our present results with the adoption of this perioperative management strategy with contemporary published benchmarks, it is clear that the outcomes, complication rates, and resource usage compare favorably. Given the differences in case distribution among centers, the results of the present study serve as a demonstration of the feasibility of this approach being extended to neonates. The lack of a suitable control group has precluded determining whether a causal relationship exists between EE and improved clinical outcomes. The generalizability of such an approach is institution dependent, because it requires broad-based support from cardiac surgery, cardiology, anesthesia, and ICU to be successfully implemented.

\section{CONCLUSIONS}

Most children undergoing congenital heart surgery can be extubated in the operating room. Most neonates, including many undergoing complex procedures, can be extubated within the first 24 hours after surgery. Implementation of an EE strategy for children with congenital heart disease is safe, feasible, and associated with good outcomes and low resource usage.

\section{References}

1. Alghamdi AA, Singh SK, Hamilton BCS, Yadava M, Holtby H, Van Arsdell GS, et al. Early extubation after pediatric cardiac surgery: systematic review, meta-analysis, and evidence-based recommendations. J Card Surg. 2010;25: 586-95.

2. Lawrence EJ, Nguyen K, Morris SA, Hollinger I, Graham DA, Jenkins KJ, et al. Economic and safety implications of introducing fast tracking in congenital heart surgery. Circ Cardiovasc Qual Outcomes. 2013;6:201-7.

3. Howard F, Brown KL, Garside V, Walker I, Elliott MJ. Fast-track paediatric cardiac surgery: the feasibility and benefits of a protocol for uncomplicated cases. Eur J Cardiothorac Surg. 2010;37:193-6.

4. Meibner U, Scharf J, Dötsch J, Schroth M. Very early extubation after open-hear surgery in children does not influence cardiac function. Pediatr Cardiol. 2008;29: 317-20.

5. Vricella LA, Dearani JA, Gundry SR, Razzouk AJ, Brauer SD, Bailey LL. Ultra fast track in elective congenital cardiac surgery. Ann Thorac Surg. 2000;69: 865-71.

6. Mittnacht AJC, Thanjan M, Srivastava S, Joashi U, Bodian C, Hossain S, et al. Extubation in the operating room after congenital heart surgery in children. J Thorac Cardiovasc Surg. 2008;136:88-93.

7. Jacobs JP. Introduction-databases and the assessment of complications associated with the treatment of patients with congenital cardiac disease. Cardiol Young. 2008;18( suppl 2):1-37.

8. Jenkins KJ, Gauvreau K, Newburger JW, Spray TL, Moller JH, Iezzoni LI. Consensus-based method for risk adjustment for surgery for congenital heart disease. J Thorac Cardiovasc Surg. 2002;123:110-8.

9. Preisman S, Lembersky H, Yusim Y, Raviv-Zilka L, Perel A, Keidan I, et al. A randomized trial of outcomes of anesthetic management directed to very early extubation after cardiac surgery in children. J Cardiothorac Vasc Anesth. 2009;23:348-57

10. Kurihara Y, Shime N, Miyazaki T, Hashimoto S, Tanaka Y. Clinical and hemodynamic factors associated with the outcome of early extubation attempts after right heart bypass surgery. Interact Cardiovasc Thorac Surg. 2009;8:624-8.

11. Schultz TR, Lin RJ, Watzman HM, Durning SM, Hales R, Woodson A, et al Weaning children from mechanical ventilation: a prospective randomized trial of protocol-directed versus physician-directed weaning. Respir Care. 2001;46: 772-82.

12. Blackwood B, Murray M, Chisakuta A, Cardwell CR, O'Halloran P Protocolized versus non-protocolized weaning for reducing the duration of invasive mechanical ventilation in critically ill paediatric patients. Cochrane Database Syst Rev. 2013;CD009082.

13. Farias J, Alía I, Retta A, Olazarri F, Fernández A, Esteban A, et al. An evaluation of extubation failure predictors in mechanically ventilated infants and children. Intensive Care Med. 2002;28:752-7.

14. Shaath GA, Jijeh A, Faruqui F, Bullard L, Mehmood A, Kabbani MS Ventilator-associated pneumonia in children after cardiac surgery. Pediatr Cardiol. 2014;35:627-31

15. Pasquali SK, Jacobs ML, He X, Shah SS, Peterson ED, Hall M, et al Variation in congenital heart surgery costs across hospitals. Pediatrics. 2014; 133:553-60.

16. Shanmugam G, Clark LL, Burton HJ, Warren AE, O'Blenes SB, Hancock Friesen CL. Improving and standardizing capture of pediatric cardiac surgical complications. J Thorac Cardiovasc Surg. 2012;144:570-6.

17. Jacobs JP, Jacobs ML, Mavroudis C, Backer CL, Lacour-Gayet FG Tchervenkov CI, et al. Nomenclature and databases for the surgical treatment of congenital cardiac disease — an updated primer and an analysis of opportunities for improvement. Cardiol Young. 2008;18(suppl 2):38-62.

18. Benavidez OJ, Gauvreau K, Nido PD, Bacha E, Jenkins KJ. Complications and risk factors for mortality during congenital heart surgery admissions. Ann Thorac Surg. 2007;84:147-55.

\section{Discussion}

Dr Ralph Mosca (New York, NY). Congratulations on an important study, which seeks to define the feasibility and utility of a clinical strategy of EE for all patients after congenital heart surgery.

EE of the vast majority of infants and children is a part of the postoperative protocol in most major cardiac centers; however, it might be particularly complicated in the neonate after 
cardiac surgery. The higher metabolic demands, a proportionately greater negative effect on cardiac and respiratory function, combined with a diminished reserve, places these patients at greater risk of failure. The caregiver must weigh the risks of prolonged extubation with the negative consequences of premature extubation. $\mathrm{EE}$ in the operating room is further encumbered by requiring transport of the patients after this important intervention.

In your study, although neonates were included, they constituted a relatively small proportion of the group, about $16 \%$. The data were also somewhat skewed toward those with lower complexity (96\% of them had a Risk Adjustment for Congenital Heart Surgery score of <3). Also, 63\% of your neonates were extubated within 24 hours; however, the reintubation rate in that group was $11.5 \%$, somewhat greater than the $4 \%$ to $6 \%$ often reported in published studies. The modes of failure and reasons for reintubation were also not well delineated and would be helpful to us as readers.

My first question is: did the patients who failed early extubation have a more prolonged second period of intubation?

Dr Harris. Thank you for the question. Those are very important points.

I think, obviously, the neonates are a more fragile population. We found that of those who had to be reintubated, 7 of the 63 , about one half of them were extubated for a second time within 24 hours of that reintubation. This might have resulted from an enhanced sensitivity to the analgesic or sedative medication they had received around the time of extubation in the neonates.

Dr Mosca. The authors also believe that protocols might be superior to objective data-driven extubation parameters. The current protocol used a subjective evaluation of the patients' readiness for extubation. The study had no control group. Patients in the EE cohort were less likely to have postoperative complications and had shorter ICU and hospital stays. However, given the nonrandomized nature of the present study, an inherent selection bias is present, making it impossible to assign causality to this relationship. Was propensity matching used to try to reduce the effects of confounding in the study?

Dr Harris. Thank you for the comments. Yes, I think an important limitation of the study is that we did not have a control group. Obviously, the children who had DE were the children who had more risk factors and were overall sicker. Therefore, it was not surprising that they had a greater complication rate and greater mortality than those who were extubated early.

We did not believe that using a retrospective control group was justifiable because a number of programmatic changes occurred that had coincided with implementation of the EE strategy at our institution. We did not perform propensity matching.

Dr Mosca. Perhaps if you do perform propensity matching to compare the groups, it would help to remove some of the selection bias and further clarify the benefits.

Finally, the published data concerning early postoperative care in neonates quotes a nearly $30 \%$ incidence of early complicating factors such as reduced lung compliance, low cardiac output, and labile pulmonary resistance. Given your experience and your predictors of delayed extubation, are there additional subgroups of patients for whom you would currently avoid EE?

I enjoyed your report, and I am sure it will provide stimulus for future studies.

Dr Harris. Thank you for the question. I did not have time to go over the predictors of DE in my presentation but they were shown in the abstract.

Right now at our institution, before the procedure, the surgeon and anesthesiologist discuss the patient's likelihood of being extubated after the procedure. The suitability for $\mathrm{EE}$ is then reviewed after the procedure, taking into account the surgical course, postoperative transesophageal echocardiographic findings, and the overall cardiorespiratory status of the patient. If patients are transported to the ICU in an intubated fashion, the need for invasive ventilator support is reassessed in a joint fashion at least twice in the first 24 hours by the cardiology, cardiac surgery, and ICU staff.

We did not have specific factors that precluded EE, but obviously, for some children, in particular, for neonates, if they have multiple risk factors, such as those shown in the present study, including preoperative mechanical ventilation, it would be unlikely that they will be extubated immediately after the procedure.

Dr Mosca. I would hope that is where future research on this topic would focus. If you just considered your DE predictors, you would rule out a fair number of complex procedures and most neonates, although you have shown that they will do pretty well anyhow. Additionally, it would be helpful to further delineate those patients who should not receive EE. For example, a patient with reduced left ventricular function after an anomalous coronary (anomalous left coronary artery from the pulmonary artery) repair, for whom extubating early could prove harmful, given the potentially adverse effects of negative respiration on the left ventricle in that subgroup.

So, great work. I think it should serve as a stimulus for future research on EE in our patients. Thank you. 